\title{
HVS 7: a chemically peculiar hyper-velocity star ${ }^{\star \star \star}$
}

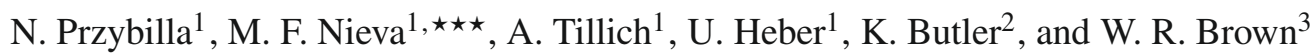 \\ 1 Dr. Remeis-Sternwarte Bamberg, Universität Erlangen-Nürnberg, Sternwartstr. 7, 96049 Bamberg, Germany \\ e-mail: przybilla@sternwarte. uni-erlangen.de \\ 2 Universitätssternwarte München, Scheinerstr. 1, 81679 München, Germany \\ 3 Smithsonian Astrophysical Observatory, 60 Garden Street, Cambridge, MA 02138, USA
}

Received 24 June 2008 / Accepted 18 July 2008

ABSTRACT

\begin{abstract}
Context. Hyper-velocity stars are suggested to originate from the dynamical interaction of binary stars with the supermassive black hole in the Galactic centre (GC), which accelerates one component of the binary to beyond the Galactic escape velocity. Aims. The evolutionary status and GC origin of the hyper-velocity star SDSS J113312.12+010824.9 (aka HVS 7) is constrained from a detailed study of its stellar parameters and chemical composition.

Methods. High-resolution spectra of HVS 7 obtained with UVES on the ESO VLT were analysed using state-of-the-art NLTE/LTE modelling techniques that can account for a chemically-peculiar composition via opacity sampling.

Results. Instead of the expected slight enrichments of $\alpha$-elements and near-solar iron, huge chemical peculiarities of all elements are apparent. The helium abundance is very low ( $<1 / 100$ solar), $\mathrm{C}, \mathrm{N}$ and $\mathrm{O}$ are below the detection limit, i.e they are underabundant $(<1 / 100, \lessgtr 1 / 3$ and $<1 / 10$ solar). Heavier elements, however, are overabundant: the iron group by a factor of $\sim 10, \mathrm{P}, \mathrm{Co}$ and $\mathrm{Cl}$ by factors $\sim 40,80$ and 440 and rare-earth elements and mercury even by $\sim 10000$. An additional finding, relevant also for other chemically peculiar stars are the large NLTE effects on abundances of Ti II and Fe II ( $\sim 0.6-0.7$ dex). The derived abundance pattern of HVS 7 is characteristic for the class of chemical peculiar magnetic B stars on the main sequence. The chemical composition and high projected rotation velocity $v \sin i=55 \pm 2 \mathrm{~km} \mathrm{~s}^{-1}$ render a low mass nature of HVS 7 as a blue horizontal branch star unlikely.

Conclusions. Such a surface abundance pattern is caused by atomic diffusion in a possibly magnetically stabilised, non-convective atmosphere. Hence all chemical information on the star's place of birth and its evolution has been washed out. High precision astrometry is the only means to validate a GC origin for HVS 7.
\end{abstract}

Key words. Galaxy: halo - stars: abundances - stars: chemically peculiar - stars: distances - stars: early-type stars: individual: SDSS J113312.12+010824.9

\section{Introduction}

Hills (1988) predicted that the tidal disruption of a binary by a supermassive black hole (SMBH) could lead to the ejection of stars with velocities exceeding the escape velocity of our Galaxy. The Galactic centre (GC) is the suspected place of origin of these so-called hyper-velocity stars (HVSs), as it hosts a SMBH. The first HVSs have only recently been discovered serendipitously (Brown et al. 2005; Hirsch et al. 2005; Edelmann et al. 2005). A systematic search for such objects has resulted in the discovery of 8 additional HVSs (see Brown et al. 2007; Heber et al. 2008a).

A determination of the space motion of a HVS can be used to trace back the trajectory unambiguously to the place of ejection. This has been done only for one HVS (HD 271791, originating from the Galactic rim, Heber et al. 2008a), as sufficiently accurate proper motions are lacking for all other HVSs at present.

Alternatively, the chemical composition of a star may be used to constrain its place of origin. Stars in the GC have a unique chemical composition, as they show enhancement of the $\alpha$-elements and roughly solar iron (e.g. Cunha et al. 2007). So

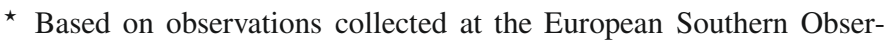
vatory, Paranal, Chile, proposal 079.D-0756(A).

$\star \star$ Appendix A is only available in electronic form at

http://www. aanda.org

$\star \star \star$ Current address: Max-Planck-Institut für Astrophysik, KarlSchwarzschild-Str. 1, 85741 Garching, Germany. far, only one object could be studied in this way (HE 0437-5439, which probably originates in the Large Magellanic Cloud, Przybilla et al. 2008). This method is currently limited by the need to obtain high-resolution spectra of faint objects.

At present, no HVS can unambiguously be related to a GC origin. Instead, alternative methods of acceleration such as dynamical ejection from dense cluster cores (Leonard 1991) or disruption of a binary by an intermediate-mass black hole (Gualandris \& Portegies Zwart 2007) challenge the SMBH paradigm.

SDSS J113312.12+010824.9 (aka HVS 7, Brown et al. 2006) is the 4th-brightest HVS candidate known $(V=17.80)$. Its spectral morphology of late B-type is consistent with either an intermediate-mass star close to the main sequence or a low-mass star on the blue horizontal branch (Brown et al. 2006; Heber et al. 2008b). We obtained high-resolution spectra of HVS 7 with UVES on the VLT and perform a quantitative spectral analysis using state-of-the-art modelling techniques in the present work to constrain its nature and its place of origin.

\section{Observations and quantitative analysis}

Seventeen spectra of HVS7 with a total integration time of $\sim 6.8 \mathrm{~h}$ were obtained between April 15 and May 10, 2007, covering the range of 3750 to $4950 \AA$ and 5700 to $9000 \AA$ at a resolving power of $R \approx 35000$. The data reduction followed 
procedures described by Koester et al. (2001). A spectrum of the DC white dwarf WD 1055-072 was also taken so that the continuum could be normalised reliably. No radial velocity variations were found within the detection limits. A peak $S / N$ of $\approx 80$ per resolution element was measured for the coadded spectrum in the blue.

An inspection of the spectrum indicated at first glance that HVS 7 is a chemically-peculiar (CP) late B-type (Bp) star with moderate (projected) rotational velocity $v \sin i$. The helium lines are extremely weak, very strong silicon lines dominate the metal line spectrum. A closer inspection identified most of the $\alpha$ process elements and most of the iron group elements in the spectrum, and many heavier species up to rare-earth elements and mercury. The presence of chlorine and strong phosphorus and cobalt lines in the spectrum of HVS 7 is unusual even for $\mathrm{CP}$ stars. Moreover, lines of carbon, nitrogen and oxygen are absent within the present detection limits. Many observed spectral lines remain unidentified, in the red spectral region, in particular.

The quantitative analysis of HVS 7 was carried out based on standard assumptions made in the field of CP stars (in particular assuming a chemically homogeneous atmosphere). In addition, we went beyond the standard by accounting for NLTE effects for many important elements. We followed the methodology discussed by Przybilla et al. (2006) and Nieva \& Przybilla (2007), with some modifications and extensions in order to account for the chemical peculiarity of the stellar atmosphere.

In brief, NLTE line-formation calculations were performed on the basis of line-blanketed LTE model atmospheres (ATLAS12, Kurucz 1996) using updated versions of DETAIL and SURFACE (Giddings 1981; Butler \& Giddings 1985). The coupled statistical equilibrium and radiative transfer problem was solved with DETAIL and SURFACE provided the formal solution based on the resulting NLTE occupation numbers. Line-blocking within DETAIL was realised here using an opacity sampling technique according to Kurucz (1996). State-of-the-art model atoms were employed as indicated in Table 1 , and in addition the hydrogen model atom of Przybilla \& Butler (2004). LTE lineformation was performed with SURFACE. The list of elements for the spectrum synthesis was extended to the "peculiar" species for the present work. See Appendix A (available online) for details of the line-formation calculations.

The chemical peculiarity of the stellar atmosphere required an iterative approach to the analysis. Starting with a model with scaled solar abundances a full analysis was performed. The resulting abundances were used to compute an improved model with ATLAS12, which indicated corrections to stellar parameters and abundances in a second step. Several such iterations were required to obtain consistency and a tailored atmospheric model.

The effective temperature $T_{\text {eff }}$ and the surface gravity $\log g$ were determined from the Stark-broadened Balmer lines $(\mathrm{H} \beta$ to $\mathrm{H}_{11}$ ) and the ionisation equilibrium of $\mathrm{Si} \mathrm{II/III} \mathrm{(the} \mathrm{strongly}$ blended Si I $23905 \AA$ line [in LTE] is also consistent). The microturbulence velocity $\xi$ was derived in the standard way by demanding that the line abundances within Fe II be independent of equivalent width. The uncertainties in the stellar parameters ( $T_{\text {eff }}, \log g$ ) were constrained by the quality of the match of the spectral indicators within the given $S / N$ limitations. Heber et al. (2008b) determined $T_{\text {eff }}=12600 \pm 500 \mathrm{~K}, \log g=3.71 \pm 0.2$ from an LTE analysis of the Balmer series in spectra of lower resolution in excellent agreement with the present results.

Elemental abundances were determined from visual fits to profiles of isolated lines (or lines with resolved blends). Spectrum synthesis was mandatory because at the given $v \sin i$ the identification of line blends has to be considered carefully
Table 1. Stellar parameters and elemental abundances of HVS 7.

\begin{tabular}{|c|c|c|c|c|c|c|}
\hline \multicolumn{2}{|c|}{$\begin{array}{l}T_{\text {eff }}(\mathrm{K}) \\
\log g(\mathrm{cgs}) \\
\xi\left(\mathrm{km} \mathrm{s}^{-1}\right) \\
v \sin i\left(\mathrm{~km} \mathrm{~s}^{-1}\right)\end{array}$} & \multicolumn{2}{|l|}{$\begin{array}{l}12000 \pm 500 \\
3.8 \pm 0.1 \\
3 \pm 1 \\
55 \pm 2\end{array}$} & \multirow{2}{*}{$\begin{array}{l}M / M_{\odot} \\
R / R_{\odot} \\
L / L_{\odot} \\
\tau_{\text {evol }}(\mathrm{Myr}) \\
\text { Ion } \\
\end{array}$} & \multicolumn{2}{|l|}{$\begin{array}{l}3.7 \pm 0.2 \\
4.0 \pm 0.1 \\
300 \pm 50 \\
150 \pm 10\end{array}$} \\
\hline Ion & $\varepsilon^{\mathrm{NLTE}}$ & $\varepsilon^{\mathrm{LTE}}$ & \# & & $\varepsilon^{\mathrm{LTE}}$ & \# \\
\hline $\mathrm{He} \mathrm{I}^{1}$ & $8.90 \pm 0.11$ & $9.17 \pm 0.11$ & 2 & Ca II & 6.60 & 1 \\
\hline $\mathrm{C} \mathrm{II}^{2}$ & $\leq 6.50$ & $\leq 6.47$ & . & Sc II & 4.00 & 1 \\
\hline $\mathrm{N} \mathrm{II}^{3}$ & $\leq 7.50$ & $\leq 7.60$ & .. & Cr II & $6.40 \pm 0.12$ & 10 \\
\hline $\mathrm{OI}^{4}$ & $\leq 7.70$ & $\leq 8.20$ & . & Mn II & $\leq 6.50$ & \\
\hline $\operatorname{Mg}$ II $^{5}$ & 6.00 & 5.80 & 1 & Co II & $6.82 \pm 0.21$ & 5 \\
\hline Si II ${ }^{6}$ & $8.69 \pm 0.13$ & $8.61 \pm 0.15$ & 12 & Sr II & 5.00 & 1 \\
\hline Si III ${ }^{6}$ & 8.65 & 8.80 & 1 & Y II & $5.02 \pm 0.18$ & 5 \\
\hline $\mathrm{S} \mathrm{II}^{7}$ & $7.35 \pm 0.12$ & $7.44 \pm 0.14$ & 2 & Eu II & $5.05 \pm 0.16$ & 3 \\
\hline $\mathrm{Ti}_{\mathrm{II}}^{8}$ & $6.20 \pm 0.11$ & $5.64 \pm 0.11$ & 7 & Gd II & $5.93 \pm 0.13$ & 4 \\
\hline $\mathrm{Fe} \mathrm{II}^{8}$ & $8.44 \pm 0.13$ & $7.78 \pm 0.16$ & 26 & Dy II & $6.00 \pm 0.23$ & 5 \\
\hline P II & $\ldots$ & $7.13 \pm 0.21$ & 2 & Dy III & $4.83 \pm 0.18$ & 3 \\
\hline $\mathrm{Cl}$ II & $\ldots$ & $7.92 \pm 0.21$ & 5 & Hg II & 4.90 & 1 \\
\hline
\end{tabular}

$\varepsilon(X)=\log (X / \mathrm{H})+12$. Error estimates consist of statistical $1 \sigma$ uncertainties derived from line-to-line scatter (\# lines) plus 0.1 dex for continuum placement uncertainty (added in quadrature); realistic uncertainties, including systematic effects e.g. if a magnetic field were present, are expected to be larger. NLTE model atoms: ${ }^{1}$ Przybilla (2005); ${ }^{2}$ Nieva \& Przybilla (2006, 2008); ${ }^{3}$ Przybilla \& Butler (2001); ${ }^{4}$ Przybilla et al. (2000); ${ }^{5}$ Przybilla et al. (2001); ${ }^{6}$ Becker \& Butler (1990), extended and updated; ${ }^{7}$ Vrancken et al. (1996), updated; ${ }^{8}$ Becker (1998).

for a successful analysis. Upper limits to the $\mathrm{C}, \mathrm{N}$ and $\mathrm{O}$ abundances were determined from C II $\lambda 4267 \AA$, N II $\lambda 3995 \AA$ and O I $\lambda \lambda 7771-5 \AA$, the strongest predicted lines for these elements. The only available $\mathrm{Hg}$ line ( $\mathrm{Hg}$ II $\lambda 3984 \AA$ ) is blended, therefore the $\mathrm{Hg}$ abundance is regarded as uncertain.

The results of the analysis are summarised in Table 1, the final synthetic spectrum is compared to observation for some exemplary regions in Fig. 1. Overall, good to excellent agreement between the model and observation is achieved. Note that many spectral lines are strongly blended and were omitted from the analysis though a good match is obtained in the comparison with the complete synthetic spectrum, with a few exceptions. In addition, several observed lines are unaccounted for by the model. No positive identification of these lines could be found, a common problem in the field of CP stars, because the atomic data are unavailable. These transitions supposedly arise from highlyexcited levels of iron group species, which are unobserved at normal abundance values, or are lines of heavier elements.

Many chemical peculiarities relative to the solar composition (Grevesse \& Sauval 1998) are found, as shown in Fig. 2. In general, we find an increase in abundance with atomic weight. The helium abundance is extremely low $(<1 / 100$ solar), $\mathrm{C}, \mathrm{N}$ and $\mathrm{O}$ are absent within the detection limits $(<1 / 100, \lesssim 1 / 3,<1 / 10$ solar, respectively) and magnesium is markedly underabundant $(\sim 1 / 30$ solar). Silicon is the most abundant metal $(>10 \times$ solar) and chlorine, cobalt and phosphorus are pronouncedly overabundant ( 440, 80 and $40 \times$ solar). All heavier elements are largely overabundant: $\sim 10 \times$ solar values for iron group elements and $\sim 10000 \times$ solar values for rare-earth elements and mercury.

NLTE effects. There are very few NLTE analyses of CP stars available in the literature, which mostly deal with $\lambda$ Boo stars (Paunzen et al. 1999; Kamp et al. 2001). We therefore want to 


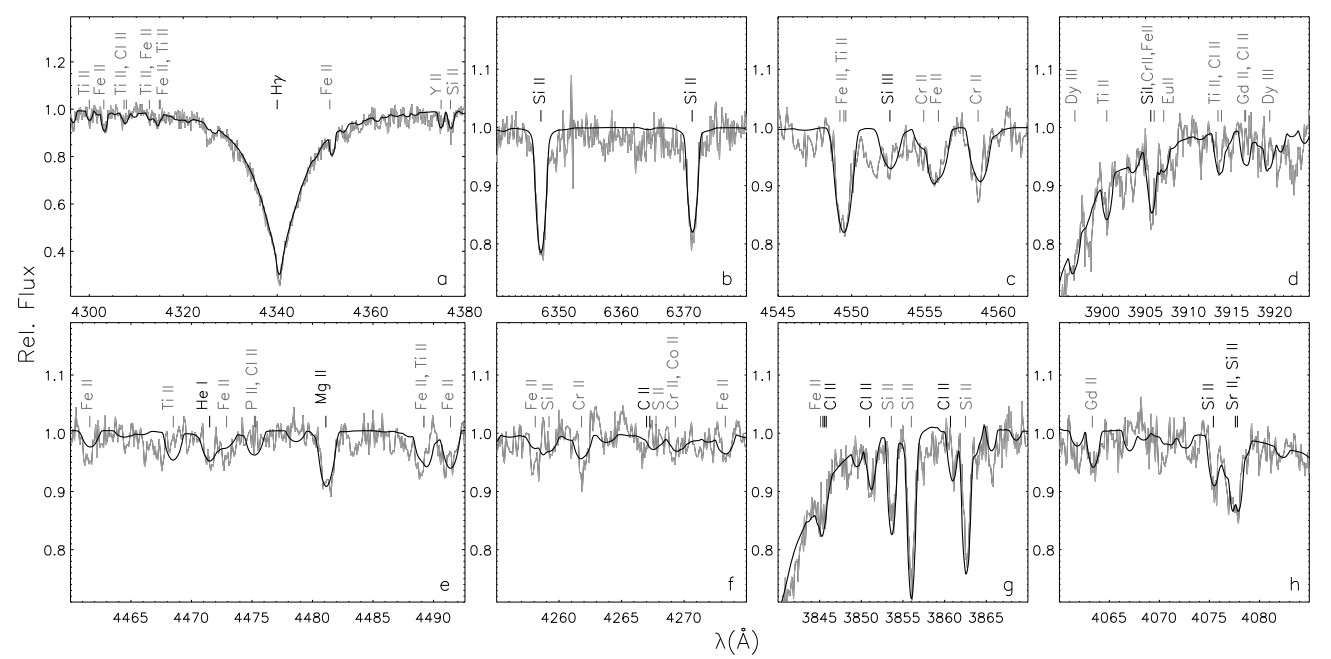

Fig. 1. Comparison of spectrum synthesis for HVS 7 (full line, best fit for abundances as in Table 1) with observations (grey), exemplary for some strategic regions. Panels a) to d) illustrate the $T_{\text {eff }}$ and $\log g$ determination from Balmer lines ( $\mathrm{H} \gamma$ in a)) and the Si ionisation equilibrium: Si II b), $\mathrm{Si}$ III c) and Si I d). Note the strong Si II lines in panel b) indicative of a Si overabundance. Panel e) illustrates the weakness (deficiency) of He I and $\mathrm{Mg}$ II, f) the absence (deficiency) of C II, g) the presence (strong enrichment) of Cl II and $\mathbf{h}$ ) a blend of Sr II and Si II. Iron group elements are highlighted in c) and rare-earth elements in d). Other prominent spectral lines are identified.

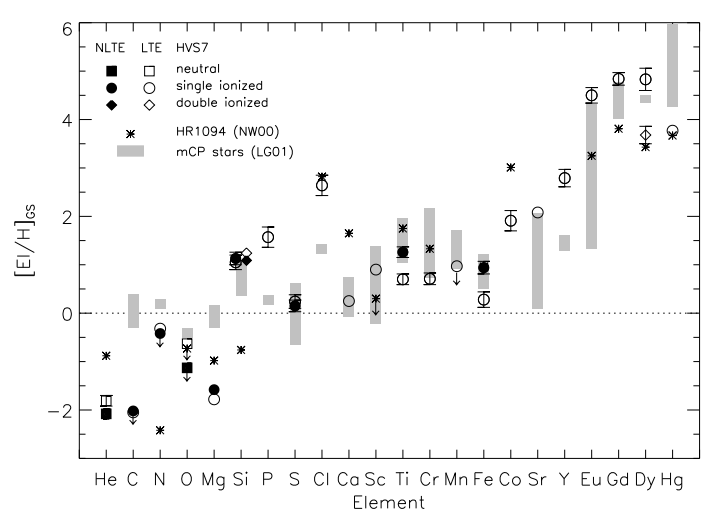

Fig. 2. Abundance pattern of HVS 7 (relative to solar values, Grevesse \& Sauval 1998) in comparison to magnetic CP stars. Symbols are identified in the legend. The error bars represent values from Table 1. Realistic errors, accounting for systematic effects (e.g. if a magnetic field were present), are expected to be larger. Upper limits are indicated by arrows. Asterisks mark abundances for the Cl-Co-rich B9p star HR 1094 (Nielsen \& Wahlgren 2000) and grey bars abundance ranges observed in a sample of magnetic CP stars (López-García et al. 2001).

briefly discuss some results that may be of relevance to other studies of Bp objects and possibly for other CP star groups.

NLTE and LTE abundances from the weak He I lines (only triplet lines are observed) show differences up to $0.3 \mathrm{dex}$ (He I $\lambda 4471 \AA$ ), as a significant NLTE overpopulation in the triplet states can build up deep in the atmosphere because radiative de-excitation to the ground state is forbidden. The same mechanism is in operation for O I $\lambda \lambda 7771-5 \AA$. Similar cases may be P II and Cl II (where we lack model atoms for NLTE calculations). Lines from spin systems which are not connected radiatively to the ground state are observed for both ions, while lines from the spin systems that include the ground state remain below the detection limit, although both should be visible when assuming LTE. NLTE effects for C II $\lambda 4267 \AA$, N II $\lambda 3995 \AA$ (for upper limit abundances), Mg II and S II remain small, as the lines are formed deep in the atmosphere where level populations are close to detailed equilibrium. The strongest metal lines in the spectrum, of Si II, are often saturated and the NLTE effects remain small because the lines become optically thick.

Differences between the NLTE and LTE analyses are mostly similar to those in main sequence stars of normal composition (Hempel \& Holweger 2003), except for Ti II and Fe II. For these, surprisingly large NLTE effects are found here, $\sim 0.6-0.7 \mathrm{dex}$, exceeding even the effects found in supergiants (Przybilla et al. 2006) at similar $T_{\text {eff }}$. The main NLTE mechanisms in these cases are an overionisation of the ground state and the energetically low levels. Because of the large overabundances the lines form at much smaller depths in the atmosphere than under normal conditions (though avoiding saturation), so that the level populations deviate strongly from detailed equilibrium values. Such a mechanism is also plausible for the first ions of the rare-earth elements, which have ionisation energies of about $10-12 \mathrm{eV}$, i.e. they are efficiently ionised by the intense radiation field longward of the Ly jump. This may e.g. explain the virtual absence of expected Gd II resonance lines in the optical, whereas lines from excited Gd II levels are observed. Accounting for NLTE effects may also help to establish the Dy II/III ionisation equilibrium.

For the moment, we can conclude that some CP stars with elemental overabundances, such as the Bp star analysed here, may be good candidates for the study of NLTE effects. Further investigations will be required, as the present results cannot be generalised to other CP star classes.

\section{Discussion}

Based on the position of HVS 7 in a $T_{\text {eff }}-\log g$-diagram alone one cannot decide whether the star is an intermediate-mass star of 3-4 $M_{\odot}$ close to the main sequence or a low-mass star of $\sim 0.5-0.7 M_{\odot}$ on the horizontal branch. Heber et al. (2008b) show the problem in their Fig. 2. An important constraint is also the rotational velocity, which is in general low $\left(<8 \mathrm{~km} \mathrm{~s}^{-1}\right)$ for hot horizontal branch stars $\left(T_{\text {eff }} \gtrsim 11000 \mathrm{~K}\right.$, Behr 2003). The high $v \sin i$ of $55 \mathrm{~km} \mathrm{~s}^{-1}$ does not necessarily rule out an HB star, as the star might have been spun up by tidal locking to the orbital motion before the binary was disrupted (see Hansen 2007) to release the HVS. Chemical peculiarities can occur both on the main sequence (e.g. Smith 1996) and on the horizontal branch 


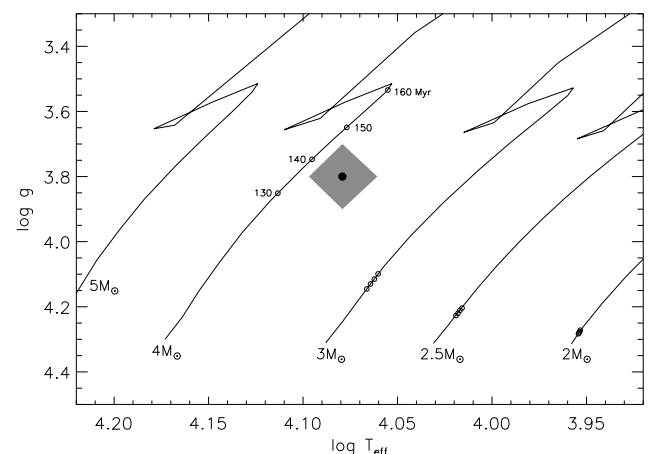

Fig. 3. Position of HVS 7 in the $T_{\text {eff }}-\log g$ diagram for the determination of its evolutionary mass and age (error box in grey). Evolutionary tracks for solar metallicity are adopted from Schaller et al. (1992). Time markers in the range 130-160 Myr are shown for each track.

(e.g. Moehler 2001; Behr 2003), though the density of metal lines in the latter case is much lower than observed for HVS 7. Hence, HVS 7 is very likely a main-sequence star.

A comparison with evolutionary tracks (Schaller et al. 1992) allows the mass of HVS 7 to be constrained (Fig. 3), and other fundamental stellar parameters such as radius $R$ and luminosity $L$, and its evolutionary lifetime $\tau_{\text {evol }}$ to be determined, see Table 1. The spectroscopic distance is then $59 \pm 6 \mathrm{kpc}$, in excellent agreement with the distance estimate of Brown et al. (2006). Note that the chemical peculiarities affect only the outer layers of the star. Moreover, they have developed with time, so that evolution tracks for standard composition are appropriate.

An identification of HVS 7 as a CP star comes not entirely unexpected in view of the commonness of late B-type stars with abundance peculiarities. In the following we attempt to classify HVS 7 within the zoo of CP stars. The dominance of the Si lines defines HVS 7 as a hot Bp star (e.g. Smith 1996), indicating a connection to magnetic stars. Indeed, the observed chemical peculiarities for the elements heavier than chlorine resemble in most cases those usually found for magnetic CP stars (e.g. the sample discussed by López-García et al. 2001), see Fig. 2. However, the lighter elements deviate in many respect from this behaviour. We therefore include also HR 1094 in the comparison, a rare member of the Cl-Co-rich stars (Nielsen \& Wahlgren 2000) with similar stellar parameters as HVS 7. Noteworthy is also the He-weakness. We conclude that HVS 7 is an exceptional member of the Bp stars, showing characteristics of several other subclasses of CP stars. The abundance peculiarities may be understood in the standard framework for the theoretical explanation of CP stars: via atomic diffusion processes, i.e. the interplay of gravitational settling and radiative levitation, in magnetically stabilised, non-convective atmospheres (e.g. Michaud 1970).

As HVS 7 might be a magnetic star, we can constrain its field strength from extra broadening of metal lines (in addition to rotation) due to the Zeeman effect. Assuming that this broadening can be mimicked by microturbulence, we derive an upper limit of $3 \mathrm{kG}$ using Eqs. (1) and (2) of Kupka et al. (1996). This may put valuable constraints on magnetic fields in the GC if HVS 7 could be proven to be of GC origin (see below) and the field as being of fossil origin (note that the star S2 near the GC is also suggested to be magnetic, Martins et al. 2008). A neglect of Zeeman splitting results in the overestimation of abundances, an effect most pronounced for the rare-earth elements. In addition, chemical surface inhomogeneities (e.g. spots) may be caused by a magnetic field. In this case the abundances derived here would be averaged surface abundances.
The aim of our spectral analysis was to search for chemical signatures that might constrain the place of birth of HVS 7 in the Galaxy, e.g. in the GC. However, diffusion processes have erased any indication of its original composition.

A verification of the Hills mechanism for the acceleration of HVS 7 has to await precise astrometry. Proper motion measurements have to be combined with the known radial velocity to obtain its space velocity vector and to reconstruct the trajectory of HVS 7 back to its place of birth. Assuming that HVS 7 originated in the GC, we can calculate the corresponding proper motion components as described by Edelmann et al. (2005), resulting in $\mu_{\alpha}=-0.53 \mathrm{mas} \mathrm{yr}^{-1}, \mu_{\delta}=-0.51 \mathrm{mas} \mathrm{yr}^{-1}$ and a space velocity of $\sim 830 \mathrm{~km} \mathrm{~s}^{-1}$. The corresponding time of flight (120 Myr) from the $\mathrm{GC}$ to its present location is in accordance with the evolutionary life time of $150 \pm 10 \mathrm{Myr}$. However, the predicted proper motion is so small that present-day instrumentation is not capable of measuring it with sufficient precision. Final conclusions can be drawn only after the GAIA mission.

Acknowledgements. We thank the staff of the ESO Paranal observatory for their support with the observations, M. Firnstein for help with the data reduction and F. Kupka for a valuable discussion on magnetic fields in CP stars. M. F. N. and A. T. gratefully acknowledge financial support by the Deutsche Forschungsgemeinschaft (grant HE 1356/45-1).

\section{References}

Becker, S. R. 1998, in Boulder-Munich II: Properties of Hot, Luminous Stars,

ed. I. D. Howarth (San Francisco: ASP), 137

Becker, S. R., \& Butler, K. 1990, A\&A, 235, 326

Behr, B. B. 2003, ApJS, 149, 67

Brown, W. R., Geller, M. J., Kenyon, S. J., \& Kurtz, M. J. 2005, ApJ, 622, L33 Brown, W. R., Geller, M. J., Kenyon, S. J., \& Kurtz, M. J. 2006, ApJ, 647, 303 Brown, W. R., Geller, M. J., Kenyon, S. J., et al. 2007, ApJ, 671, 1708

Butler, K., \& Giddings, J. R. 1985, in Newsletter of Analysis of Astronomical Spectra, Univ. London, 9

Cunha, K., Sellgren, K., Smith, V. V., et al. 2007, ApJ, 669, 1011

Edelmann, H., Napiwotzki, R., Heber, U., et al. 2005, ApJ, 634, L181 (E05)

Giddings, J. R. 1981, Ph.D. Thesis, University of London

Grevesse, N., \& Sauval, A. J. 1998, Space Sci. Rev., 85, 161

Gualandris, A., \& Portegies Zwart, S. 2007, MNRAS, 376, L29

Hansen, B. 2007, ApJ, 671, L133

Heber, U., Edelmann, H., Napiwotzki, R., et al. 2008a, A\&A, 483, L21

Heber, U., et al. 2008b, in Hot Subdwarf Stars and Related Objects, ed. U. Heber,

C. S. Jeffery, \& R. Napiwotzki (San Francisco: ASP), 167

Hempel, M., \& Holweger, H. 2003, A\&A, 408, 1065

Hills, J. G. 1988, Nature, 331, 687

Hirsch, H. A., Heber, U., O’Toole, S. J., \& Bresolin, F. 2005, A\&A, 444, L61

Kamp, I., Iliev, I. K., Paunzen, E., et al. 2001, A\&A, 375, 899

Koester, D., Napiwotzki, R., Christlieb, N., et al. 2001, A\&A, 378, 556

Kupka, F., Ryabchikova, T. A., Weiss, W. W., et al. 1996, A\&A, 308, 886

Kurucz, R. L. 1996, in Model Atmospheres and Spectrum Synthesis, ed. S. J.

Adelman, F. Kupka, \& W. W. Weiss (San Francisco: ASP), 160

Kurucz, R. L., \& Bell, B. 1995, Kurucz CD-ROM 23, SAO, Cambridge, Mass.

Leonard, P. J. T. 1991, AJ, 101, 562

López-García, Z., Adelman, S. J., \& Pintado, O. I. 2001, A\&A, 367, 859 (LG01)

Martins, F., Gillessen, S., Eisenhauer, F., et al. 2008, ApJ, 672, L119

Michaud, G. 1970, ApJ, 160, 641

Moehler, S. 2001, PASP, 113, 1162

Nielsen, K., \& Wahlgren, G. M. 2000, A\&A, 356, 146 (NW00)

Nieva, M. F., \& Przybilla, N. 2006, ApJ, 639, L39

Nieva, M. F., \& Przybilla, N. 2007, A\&A, 467, 295

Nieva, M. F., \& Przybilla, N. 2008, A\&A, 481, 199

Paunzen, E., Kamp, I., Iliev, I. K., et al. 1999, A\&A, 345, 597

Przybilla, N. 2005, A\&A, 443, 293

Przybilla, N., \& Butler, K. 2001, A\&A, 379, 955

Przybilla, N., \& Butler, K. 2004, ApJ, 609, 1181

Przybilla, N., Butler, K., Becker, S. R., et al. 2000, A\&A, 359, 1085

Przybilla, N., Butler, K., Becker, S. R., \& Kudritzki, R. P. 2001, A\&A, 369, 1009 Przybilla, N., Butler, K., Becker, S. R., \& Kudritzki R. P. 2006, A\&A, 445, 1099 Przybilla, N., Nieva, M. F., Heber, U., et al. 2008, A\&A, 480, L37

Schaller, G., Schaerer, D., Meynet, G., \& Maeder, A. 1992, A\&AS, 96, 269

Smith, K. C. 1996, Ap\&SS, 237, 77

Vrancken, M., Butler, K., \& Becker, S. R. 1996, A\&A, 311, 661 


\section{Appendix A: Spectral line analysis}

In this appendix we provide details on our spectral line analysis of HVS 7. Table A.1 summarises our line data and the results from the abundance analysis of individual lines. The first columns give the wavelength $\lambda$ (in $\AA$ ), excitation energy of the lower level $\chi$ (in $\mathrm{eV}$ ), adopted oscillator strength $\log g f$, an accuracy flag for the oscillator strength, the source of the $\log g f$ value and the reference for Stark broadening parameters (tabulations by Schöning \& Butler based on the theory of Vidal et al. 1973, were used for the Balmer lines). Then, the derived abundances $\varepsilon=\log (X / \mathrm{H})+12$ in NLTE and LTE are tabulated.

Radiative damping constants were preferentially calculated from level lifetimes, adopted from the Opacity Project (Seaton et al. 1994) and other sources in the (astro-)physics literature, where available. In other cases data were adopted from Kurucz $\&$ Bell (1995) or calculated assuming classical damping. Note that radiative damping is negligible in the present case, such that we omit a more detailed discussion. Van-der-Waals broadening was neglected, as the atmospheric plasma is well ionised throughout the line-formation region. Resonance broadening for hydrogen was also neglected for the same reason.

Finally, note that isotopic and hyperfine-structure for Eu II and $\mathrm{Hg}$ II lines were treated according to Lawler et al. (2001) and Castelli \& Hubrig (2004), respectively.

\section{Online References}

Barnard, A. J., Cooper, L., \& Shamey, L. J. 1969, A\&A, 1, 28

Bates, D., \& Damgaard, A. 1949, Phil. Trans. R. Soc. London, Ser. A, 242, 101 Biémont, E., \& Lowe, R. M. 1993, A\&A, 273, 665

Castelli, F., \& Hubrig, S. 2004, A\&A, 425, 263

Cowley, C. 1971, Observatory, 91, 139

Den Hartog, E. A., Lawler, J. E., Sneden, C., \& Cowan, J. J. 2006, ApJS, 167, 292

Fernley, J. A., Taylor, K. T., \& Seaton, M. J. 1987, J. Phys. B, 20, 6457

Froese Fischer, C., Tachiev, G., \& Irimia, A. 2006, At. Data Nucl. Data Tables, 92,607

Fuhr, J. R., \& Wiese, W. L. 1998, in CRC Handbook of Chemistry and Physics, 79th edn., ed. D. R. Lide (Boca Raton: CRC Press)

Fuhr, J. R., Martin, G. A., \& Wiese, W. L. 1988, J. Phys. \& Chem. Ref. Data, 17, Suppl. 4

Griem, H. R. 1964, Plasma Spectroscopy (New York: McGraw-Hill Book Company)

Griem, H. R. 1974, Spectral Line Broadening by Plasmas (New York, London: Academic Press)

Hannaford, P., Lowe, R. M., Grevesse, N., Biémont, E., \& Whaling, W. 1982, ApJ, 261, 736

Kurucz, R. L. 1988, in Trans. IAU XXB, ed. M. McNally (Dordrecht: Kluwer), 168

Kurucz, R. L. 1994a, Kurucz CD-ROM 20, SAO, Cambridge, Mass.

Kurucz, R. L. 1994b, Kurucz CD-ROM 22, SAO, Cambridge, Mass.

Kurucz, R. L., \& Bell, B. 1995, Kurucz CD-ROM 23, SAO, Cambridge, Mass.

Lanz, T., Dimitrijević, M. S., \& Artru, M.-C. 1988, A\&A, 192, 249

Lawler, J. E., Wickliffe, M. E., den Hartog, E. A., \& Sneden, C. 2001, ApJ, 563, 1075

Martin, G. A., Fuhr, J. R., \& Wiese, W. L. 1988, J. Phys. \& Chem. Ref. Data, 17, Suppl. 3

Matheron, P., Escarguel, A., Redon, R., Lesage, A., \& Richou, J. 2001, J. Quant. Spectrosc. Radiat. Transfer, 69, 535

Mendoza, C., Eissner, W., Le Dourneuf, M., \& Zeippen, C. J. 1995, J. Phys. B, 28,3485

Nahar, S. N. 1998, At. Data Nucl. Data Tables, 68, 183
Seaton, M. J., Yan, Y., Mihalas, D., \& Pradhan, A. K. 1994, MNRAS, 266, 805 Shamey, L. J. 1969, Ph.D. Thesis, University of Colorado

Vidal, C. R., Cooper, J., \& Smith, E. W. 1973, ApJS, 25, 37

Wiese, W. L., Smith, M. W., \& Miles, B. M. 1969, Nat. Stand. Ref. Data Ser., Nat. Bur. Stand. (US), NSRDS-NBS 22, Vol. II

Wiese, W. L., Fuhr, J. R., \& Deters, T. M. 1996, J. Phys. \& Chem. Ref. Data, Mon. 7

Younger, S. M., Fuhr, J. R., Martin, G. A., \& Wiese, W. L. 1978, J. Phys. \& Chem. Ref. Data, 7, 495

Zhang, Z. G., Svanberg, S., Palmeri, P., Quinet, P., \& Biémont, E. 2002, MNRAS,

334,1 
N. Przybilla et al.: HVS 7: a chemically peculiar hyper-velocity star, Online Material $p 2$

Table A.1. Spectral line analysis

\begin{tabular}{|c|c|c|c|c|c|c|c|}
\hline$\lambda(\AA)$ & $\chi(\mathrm{eV})$ & $\log g f$ & Acc. & Src. & Broad. & $\varepsilon^{\mathrm{NLTE}}$ & $\varepsilon^{\mathrm{LTE}}$ \\
\hline \multicolumn{8}{|l|}{ He I: } \\
\hline 4026.18 & 20.96 & -2.60 & A & FTS & $\mathrm{S}$ & 8.90 & 9.13 \\
\hline 4026.19 & 20.96 & -0.63 & A & FTS & $\mathrm{S}$ & & \\
\hline 4026.20 & 20.96 & -0.85 & A & FTS & S & & \\
\hline 4026.36 & 20.96 & -1.33 & A & FTS & S & & \\
\hline 4471.47 & 20.96 & -0.21 & A & FTS & BCS & 8.90 & 9.20 \\
\hline 4471.49 & 20.96 & -0.43 & A & FTS & BCS & & \\
\hline 4471.68 & 20.96 & -0.91 & A & FTS & BCS & & \\
\hline \multicolumn{8}{|l|}{ C II: } \\
\hline 4267.000 & 18.04 & 0.56 & $\mathrm{C}+$ & WFD & G & $\leq 6.50$ & $\leq 6.47$ \\
\hline 4267.260 & 18.04 & 0.74 & $\mathrm{C}+$ & WFD & G & & \\
\hline \multicolumn{8}{|l|}{ N II: } \\
\hline $\begin{array}{l}3995.00 \\
\text { O I: }\end{array}$ & \multicolumn{6}{|c|}{ O I: } & $\leq 7.60$ \\
\hline 7771.94 & 9.15 & 0.37 & A & WFD & $\mathrm{C}$ & $\leq 7.70$ & $\leq 8.20$ \\
\hline 7774.17 & 9.15 & 0.22 & A & WFD & $\mathrm{C}$ & & \\
\hline 7775.39 & 9.15 & 0.00 & A & WFD & $\mathrm{C}$ & & \\
\hline \multicolumn{8}{|l|}{ Mg II: } \\
\hline 4481.126 & 8.86 & 0.73 & B & FW & G & 6.00 & 5.80 \\
\hline 4481.150 & 8.86 & -0.57 & B & FW & $\mathrm{G}$ & & \\
\hline 4481.325 & 8.86 & 0.57 & B & FW & G & & \\
\hline \multicolumn{8}{|l|}{ Si II: } \\
\hline 3853.665 & 6.86 & -1.40 & $\mathrm{C}+$ & FFTI & LDA & 8.50 & 8.48 \\
\hline 3856.018 & 6.86 & -0.45 & $\mathrm{C}+$ & FFTI & LDA & 8.60 & 8.60 \\
\hline 3862.595 & 6.86 & -0.71 & $\mathrm{C}+$ & FFTI & LDA & 8.60 & 8.60 \\
\hline 3954.300 & 12.53 & -1.11 & $\mathrm{E}$ & N98 & C & 8.75 & 8.63 \\
\hline 3954.504 & 12.53 & -0.94 & $\mathrm{E}$ & N98 & $\mathrm{C}$ & & \\
\hline 4075.452 & 9.84 & -1.40 & $\mathrm{C}+$ & MER & $\mathrm{C}$ & 8.70 & 8.48 \\
\hline 4076.780 & 9.84 & -1.70 & $\mathrm{C}+$ & MER & $\mathrm{C}$ & & \\
\hline 4200.658 & 12.53 & -0.89 & $\mathrm{E}$ & N98 & $\mathrm{C}$ & 8.70 & 8.54 \\
\hline 4200.887 & 12.53 & -2.03 & E & N98 & $\mathrm{C}$ & & \\
\hline 4200.898 & 12.53 & -0.73 & D & N98 & $\mathrm{C}$ & & \\
\hline 4376.969 & 12.84 & -0.89 & $\mathrm{E}$ & N98 & $\mathrm{C}$ & 8.80 & 8.66 \\
\hline 4376.994 & 12.84 & -1.02 & E & N98 & $\mathrm{C}$ & & \\
\hline 4621.418 & 12.53 & -0.61 & $\mathrm{D}$ & MELZ & $\mathrm{C}$ & 8.80 & 8.59 \\
\hline 4621.696 & 12.53 & -1.75 & E & MELZ & $\mathrm{C}$ & & \\
\hline 4621.722 & 12.53 & -0.45 & D & MELZ & $\mathrm{C}$ & & \\
\hline 5957.561 & 10.07 & -0.35 & $\mathrm{D}$ & WSM & LDA & 8.70 & 8.52 \\
\hline 5978.929 & 10.07 & -0.06 & D & WSM & LDA & 8.70 & 8.56 \\
\hline 6347.103 & 8.12 & 0.17 & $\mathrm{C}+$ & FFTI & LDA & 8.70 & 8.82 \\
\hline 6371.359 & 8.12 & -0.13 & $\mathrm{C}+$ & FFTI & LDA & 8.75 & 8.80 \\
\hline \multicolumn{8}{|l|}{ Si III: } \\
\hline 4552.622 & 19.02 & 0.29 & $\mathrm{~B}+$ & FFTI & $\mathrm{C}$ & 8.65 & 8.80 \\
\hline \multicolumn{8}{|l|}{ P II: } \\
\hline 4420.712 & 11.02 & -0.33 & $\mathrm{D}$ & CA & $\mathrm{C}$ & $\cdots$ & 7.00 \\
\hline 4499.230 & 13.38 & 0.47 & $\mathrm{D}$ & $\mathrm{CA}$ & $\mathrm{C}$ & $\ldots$ & 7.25 \\
\hline \multicolumn{8}{|l|}{ S II: } \\
\hline 4524.675 & 15.07 & -0.94 & $\mathrm{D}$ & FW & $\mathrm{C}$ & 7.30 & 7.37 \\
\hline 4524.941 & 15.07 & 0.17 & $\mathrm{D}$ & FW & $\mathrm{C}$ & & \\
\hline 4815.552 & 13.67 & 0.09 & D & FW & $\mathrm{C}$ & 7.40 & 7.50 \\
\hline \multicolumn{8}{|l|}{ Cl II: } \\
\hline 3845.362 & 15.95 & 0.02 & D & WSM & $\mathrm{C}$ & $\ldots$ & 8.00 \\
\hline 3845.639 & 15.95 & 0.12 & $\mathrm{D}$ & WSM & $\mathrm{C}$ & & \\
\hline 3845.788 & 15.95 & -0.22 & $\mathrm{D}$ & WSM & $\mathrm{C}$ & & \\
\hline 3850.988 & 15.95 & 0.45 & $\mathrm{D}$ & WSM & $\mathrm{C}$ & $\ldots$ & 8.00 \\
\hline 3851.374 & 15.95 & 0.25 & $\mathrm{D}$ & WSM & $\mathrm{C}$ & & \\
\hline 3851.651 & 15.95 & -0.35 & $\mathrm{D}$ & WSM & $\mathrm{C}$ & & \\
\hline 3860.828 & 15.96 & 0.74 & $\mathrm{D}$ & WSM & $\mathrm{C}$ & $\ldots$ & 7.60 \\
\hline 3860.990 & 15.96 & 0.14 & $\mathrm{D}$ & WSM & $\mathrm{C}$ & & \\
\hline 3861.378 & 15.96 & -0.70 & $\mathrm{D}$ & WSM & $\mathrm{C}$ & & \\
\hline 4794.556 & 13.38 & 0.40 & $\mathrm{C}$ & FW & $\mathrm{C}$ & $\ldots$ & 7.90 \\
\hline 4810.070 & 13.38 & 0.24 & $\mathrm{C}$ & FW & $\mathrm{C}$ & $\ldots$ & 8.10 \\
\hline \multicolumn{8}{|l|}{ Ca II: } \\
\hline 3933.663 & 0.00 & 0.14 & $\mathrm{C}$ & FW & G & $\ldots$ & 6.60 \\
\hline \multicolumn{8}{|l|}{ Sc II: } \\
\hline 4246.822 & 0.32 & 0.28 & $\mathrm{D}$ & MFW & $\mathrm{C}$ & $\ldots$ & 4.00 \\
\hline
\end{tabular}


N. Przybilla et al.: HVS 7: a chemically peculiar hyper-velocity star, Online Material p 3

Table A.1. continued.

\begin{tabular}{|c|c|c|c|c|c|c|c|}
\hline$\lambda(\AA)$ & $\chi(\mathrm{eV})$ & $\log g f$ & Acc. & Src. & Broad. & $\varepsilon^{\mathrm{NLTE}}$ & $\varepsilon^{\mathrm{LTE}}$ \\
\hline \multicolumn{8}{|l|}{ Ti II: } \\
\hline 3900.551 & 1.13 & -0.45 & D & MFW & $\mathrm{C}$ & 6.20 & 5.61 \\
\hline 3913.468 & 1.12 & -0.53 & $\mathrm{D}$ & MFW & $\mathrm{C}$ & 6.10 & 5.55 \\
\hline 4300.049 & 1.18 & -0.77 & D- & MFW & $\mathrm{C}$ & 6.20 & 5.63 \\
\hline 4301.914 & 1.16 & -1.16 & D- & MFW & $\mathrm{C}$ & 6.20 & 5.67 \\
\hline 4395.033 & 1.08 & -0.66 & D- & MFW & $\mathrm{C}$ & 6.20 & 5.65 \\
\hline 4443.794 & 1.08 & -0.70 & D- & MFW & $\mathrm{C}$ & 6.20 & 5.65 \\
\hline 4911.193 & 3.12 & -0.34 & $\mathrm{D}$ & MFW & $\mathrm{C}$ & 6.30 & 5.75 \\
\hline \multicolumn{8}{|l|}{ Cr II: } \\
\hline 4110.990 & 3.76 & -2.02 & $X$ & K88 & $\mathrm{C}$ & $\ldots$ & 6.50 \\
\hline 4111.003 & 3.10 & -1.92 & $X$ & K88 & $\mathrm{C}$ & & \\
\hline 4242.364 & 3.87 & -1.33 & $X$ & YFMW & $\mathrm{C}$ & $\ldots$ & 6.50 \\
\hline 4275.567 & 3.86 & -1.71 & $X$ & K88 & $\mathrm{C}$ & $\ldots$ & 6.30 \\
\hline 4558.650 & 4.07 & -0.66 & $\mathrm{D}$ & MFW & $\mathrm{C}$ & $\ldots$ & 6.40 \\
\hline 4588.199 & 4.07 & -0.63 & $\mathrm{D}$ & MFW & $\mathrm{C}$ & $\ldots$ & 6.40 \\
\hline 4616.629 & 4.07 & -1.29 & D & MFW & $\mathrm{C}$ & $\ldots$ & 6.40 \\
\hline 4618.803 & 4.07 & -1.11 & D & MFW & $\mathrm{C}$ & $\ldots$ & 6.40 \\
\hline 4634.070 & 4.07 & -1.24 & $\mathrm{D}$ & MFW & $\mathrm{C}$ & $\ldots$ & 6.40 \\
\hline 4824.127 & 3.87 & -0.96 & $X$ & K94a & $\mathrm{C}$ & $\ldots$ & 6.30 \\
\hline 4876.399 & 3.86 & -1.47 & $\mathrm{D}$ & MFW & $\mathrm{C}$ & $\ldots$ & 6.35 \\
\hline \multicolumn{8}{|l|}{ Mn II: } \\
\hline 4755.727 & 5.40 & -1.24 & $X$ & K88 & $\mathrm{C}$ & $\ldots$ & $\leq 6.50$ \\
\hline \multicolumn{8}{|l|}{ Fe II: } \\
\hline 3863.985 & 4.15 & -3.43 & $X$ & K88 & $\mathrm{C}$ & 8.50 & 7.95 \\
\hline 3872.766 & 2.70 & -3.32 & $X$ & K88 & $\mathrm{C}$ & 8.40 & 7.80 \\
\hline 3935.962 & 5.57 & -1.86 & $\mathrm{D}$ & FMW & $\mathrm{C}$ & 8.50 & 7.92 \\
\hline 4004.080 & 10.68 & -3.72 & $X$ & K88 & $\mathrm{C}$ & 8.40 & 7.80 \\
\hline 4173.461 & 2.58 & -2.18 & $\mathrm{C}$ & FMW & $\mathrm{C}$ & 8.45 & 7.65 \\
\hline 4178.862 & 2.58 & -2.47 & $\mathrm{C}$ & FMW & $\mathrm{C}$ & 8.40 & 7.65 \\
\hline 4273.326 & 2.70 & -3.34 & $\mathrm{D}$ & FMW & $\mathrm{C}$ & 8.50 & 7.90 \\
\hline 4303.176 & 2.70 & -2.49 & $\mathrm{C}$ & FMW & $\mathrm{C}$ & 8.45 & 7.65 \\
\hline 4351.768 & 2.70 & -2.10 & $\mathrm{C}$ & FMW & $\mathrm{C}$ & 8.20 & 7.40 \\
\hline 4385.387 & 2.78 & -2.57 & $\mathrm{D}$ & FMW & $\mathrm{C}$ & 8.40 & 7.70 \\
\hline 4489.183 & 2.83 & -2.97 & $\mathrm{D}$ & FMW & $\mathrm{C}$ & 8.50 & 7.85 \\
\hline 4491.405 & 2.86 & -2.69 & $\mathrm{C}$ & FMW & $\mathrm{C}$ & 8.40 & 7.70 \\
\hline 4508.288 & 2.86 & -2.30 & $X$ & K94b & $\mathrm{C}$ & 8.40 & 7.70 \\
\hline 4520.224 & 2.81 & -2.61 & D & FMW & $\mathrm{C}$ & 8.45 & 7.70 \\
\hline 4522.634 & 2.84 & -2.11 & $X$ & K94b & $\mathrm{C}$ & 8.50 & 7.75 \\
\hline 4549.474 & 2.83 & -1.75 & $\mathrm{C}$ & FMW & $\mathrm{C}$ & 8.60 & 7.85 \\
\hline 4555.893 & 2.83 & -2.33 & $X$ & K94b & $\mathrm{C}$ & 8.40 & 7.65 \\
\hline 4576.340 & 2.84 & -3.04 & $\mathrm{D}$ & FMW & $\mathrm{C}$ & 8.40 & 7.77 \\
\hline 4629.339 & 2.81 & -2.38 & $\mathrm{D}$ & FMW & $\mathrm{C}$ & 8.30 & 7.60 \\
\hline 4635.316 & 5.96 & -1.65 & $\mathrm{D}$ & FMW & $\mathrm{C}$ & 8.40 & 7.83 \\
\hline 4666.758 & 2.83 & -3.34 & D & FMW & $\mathrm{C}$ & 8.60 & 8.00 \\
\hline 4731.453 & 2.89 & -3.37 & $\mathrm{D}$ & FMW & $\mathrm{C}$ & 8.50 & 7.90 \\
\hline 4923.927 & 2.89 & -1.32 & $\mathrm{C}$ & FMW & $\mathrm{C}$ & 8.45 & 7.80 \\
\hline 6317.983 & 7.47 & -1.99 & $X$ & K88 & $\mathrm{C}$ & 8.35 & 7.80 \\
\hline 6516.081 & 2.89 & -3.45 & $\mathrm{D}$ & FMW & $\mathrm{C}$ & 8.50 & 7.90 \\
\hline 6518.774 & 12.89 & 0.35 & $X$ & K88 & $\mathrm{C}$ & 8.40 & 7.95 \\
\hline \multicolumn{8}{|l|}{ Co II: } \\
\hline 4160.673 & 3.41 & -1.83 & $X$ & K88 & $\mathrm{C}$ & $\ldots$ & 6.50 \\
\hline 4497.431 & 3.41 & -2.54 & $X$ & K88 & $\mathrm{C}$ & $\ldots$ & 6.90 \\
\hline 4516.633 & 3.46 & -2.56 & $X$ & K88 & $\mathrm{C}$ & $\ldots$ & 6.90 \\
\hline 4660.656 & 3.36 & -2.21 & $X$ & K88 & $\mathrm{C}$ & $\ldots$ & 6.90 \\
\hline 4831.242 & 5.05 & -1.67 & $X$ & K88 & $\mathrm{C}$ & $\ldots$ & 6.90 \\
\hline \multicolumn{8}{|l|}{ Sr II: } \\
\hline 4215.520 & 0.00 & -0.17 & $X$ & FW & $\mathrm{C}$ & $\ldots$ & 5.00 \\
\hline \multicolumn{8}{|l|}{ Y II: } \\
\hline 3950.356 & 0.10 & -0.49 & $X$ & HLG & $\mathrm{C}$ & $\ldots$ & 5.00 \\
\hline 4177.636 & 0.41 & -0.16 & $X$ & HLG & $\mathrm{C}$ & $\ldots$ & 5.10 \\
\hline 4374.946 & 0.41 & 0.16 & $X$ & HLG & $\mathrm{C}$ & $\ldots$ & 4.80 \\
\hline 4398.010 & 0.13 & -1.00 & $X$ & HLG & $\mathrm{C}$ & $\ldots$ & 5.00 \\
\hline 4900.110 & 1.03 & -0.09 & $X$ & HLG & $\mathrm{C}$ & $\ldots$ & 5.20 \\
\hline \multicolumn{8}{|l|}{ Eu II: } \\
\hline 3819.67 & 0.00 & 0.51 & $\mathrm{X}$ & LWDS & C & $\cdots$ & 4.90 \\
\hline 3907.11 & 0.21 & 0.17 & $X$ & LWDS & $\mathrm{C}$ & $\ldots$ & 5.10 \\
\hline 4205.04 & 0.00 & 0.21 & $X$ & LWDS & $\mathrm{C}$ & $\ldots$ & 5.15 \\
\hline
\end{tabular}


Table A.1. continued.

\begin{tabular}{lrrlcrcc}
\hline \hline$\lambda(\AA)$ & $\chi(\mathrm{eV})$ & $\log g f$ & Acc. & Src. & Broad. & $\varepsilon^{\text {NLTE }}$ & $\varepsilon^{\text {LTE }}$ \\
\hline Gd II: & & & & & & & \\
3916.509 & 0.60 & -0.04 & $\mathrm{X}$ & DLSC & $\mathrm{C}$ & $\ldots$ & 5.80 \\
4037.323 & 0.66 & -0.11 & $\mathrm{X}$ & DLSC & $\mathrm{C}$ & $\ldots$ & 5.95 \\
4037.893 & 0.56 & -0.42 & $\mathrm{X}$ & DLSC & $\mathrm{C}$ & & \\
4063.384 & 0.99 & 0.33 & $\mathrm{X}$ & DLSC & $\mathrm{C}$ & $\ldots$ & 5.95 \\
4184.258 & 0.49 & 0.00 & $\mathrm{X}$ & DLSC & $\mathrm{C}$ & $\ldots$ & 6.00 \\
Dy II: & & & & & & & \\
3872.11 & 0.00 & 0.00 & $\mathrm{X}$ & $\mathrm{BL}$ & $\mathrm{C}$ & $\ldots$ & 5.70 \\
3944.68 & 0.00 & 0.10 & $\mathrm{X}$ & $\mathrm{BL}$ & $\mathrm{C}$ & $\ldots$ & 6.00 \\
3996.69 & 0.59 & -0.20 & $\mathrm{X}$ & $\mathrm{BL}$ & $\mathrm{C}$ & $\ldots$ & 6.00 \\
4000.45 & 0.10 & 0.06 & $\mathrm{X}$ & $\mathrm{BL}$ & $\mathrm{C}$ & $\ldots$ & 6.00 \\
4050.56 & 0.59 & -0.42 & $\mathrm{X}$ & $\mathrm{BL}$ & $\mathrm{C}$ & $\cdots$ & 6.30 \\
Dy III: & & & & & & & \\
3896.817 & 0.52 & -1.10 & $\mathrm{X}$ & $\mathrm{ZSP}$ & $\mathrm{C}$ & $\ldots$ & 4.80 \\
3919.398 & 0.52 & -1.34 & $\mathrm{X}$ & $\mathrm{ZSP}$ & $\mathrm{C}$ & $\ldots$ & 4.70 \\
3930.632 & 0.00 & -0.88 & $\mathrm{X}$ & $\mathrm{ZSP}$ & $\mathrm{C}$ & $\cdots$ & 5.00 \\
Hg II: & & & & & & & \\
3983.93 & 4.40 & -1.52 & $\mathrm{X}$ & $\mathrm{CH}$ & $\mathrm{C}$ & $\ldots$ & 4.90 \\
\hline
\end{tabular}

Accuracy indicators - uncertainties within: A: 3\%; B: 10\%; C: 25\%; D: 50\%; E: larger than 50\%; X: unknown sources of $g f$-values - BL: Biémont \& Lowe (1993); CA: Coulomb approximation (Bates \& Damgaard 1949); CH: Castelli \& Hubrig (2004); DLSC: Den Hartog et al. (2006); FFTI: Froese Fischer et al. (2006); FTS: Fernley et al. (1987); FMW: Fuhr et al. (1988); FW: Fuhr \& Wiese (1998); HLG: Hannaford et al. (1982); K88: Kurucz (1988); K94a: Kurucz (1994a); K94b: Kurucz (1994b); LWDS: Lawler et al. (2001); MELZ: Mendoza et al. (1995); MER: Matheron et al. (2001); MFW: Martin et al. (1988); N98: Nahar (1998); WFD: Wiese et al. (1996); WSM: Wiese et al. (1969), when available replaced by improved $g f$-values from Fuhr \& Wiese (1998); YFMW: Younger et al. (1978); ZSP: Zhang et al. (2002).

Sources for Stark broadening parameters - BCS: Barnard et al. (1969); C: Cowley (1971); G: Griem (1964, 1974); LDA: Lanz et al. (1988); S: Shamey (1969). 